\title{
Antiresorptive medication in oncology: the clinical and dental profile of patients in a reference center
}

\author{
Vitor Hugo Candido Ferreira ${ }^{1,2}$, Aristilia Tahara Kemp ${ }^{1}$, Joana Vendruscolo², \\ Laurindo Moacir Sassi ${ }^{2}$, Juliana Lucena Schussel ${ }^{1}$ \\ ${ }^{I}$ Department of Stomatology, Federal University of Paraná, \\ ${ }^{2}$ Department of Oral and Maxillofacial Surgery, Erasto Gaertner Cancer Center, Curitiba, Brazil
}

\begin{abstract}
J Korean Assoc Oral Maxillofac Surg 2021;47:20-24)
Objectives: The aim of this study was to evaluate the profile of patients on antiresorptive therapies for cancer treatment and assess presence of oral lesions, oral hygiene status, and knowledge regarding medication-related osteonecrosis of the jaw (MRONJ).

Materials and Methods: This was an observational cross-sectional study that evaluated patients treated with antiresorptive medication at a single cancer hospital. Clinical data were collected and oral examination was performed to assess patient oral health.

Results: From July 2017 to December 2018, 90 patients were assessed; 64 were female and 26 were male, and the mean age was 61 years. The most common drug was an intravenous bisphosphonate, zoledronic acid. Among the 90 patients, 47 presented with some type of oral disease, isolated or associated. Among these 47 patients, 9 patients (10\%) developed osteonecrosis. Oral hygiene was evaluated, and most patients, with or without MRONJ, presented with regular to poor condition. Regarding patient knowledge of the risks of MRONJ and the risks associated with dental surgery, $60 \%$ stated that they were not aware of the risks.

Conclusion: Identifying the profile of patients and their needs facilitates not only the preventive process, but also the emergence of new therapeutic options. Our study shows that most patients are weakened both by metastatic disease and antineoplastic treatment as well as by issues associated with aging because most were over 60 years of age. Collectively, this information should be considered for management of preventive and therapeutic measures.
\end{abstract}

Key words: Osteonecrosis of the jaws, Medication-related osteonecrosis of the jaw, Antiresorptives

\section{Introduction}

Antiresorptive medication is widely used for bone-related diseases in patients with osteoporosis and osteopenia, cancer metastasis, or multiple myeloma ${ }^{1}$.

In oncology, antiresorptive medication is used as adjunctive treatment to manage and limit tumor evolution. The medication acts directly on skeletal-related events associated with bone metastasis of solid tumors such as breast, prostate, and

\section{Juliana Lucena Schussel}

Department of Stomatology, Federal University of Paraná, Av. Pref. Lothário Meissner, 632, Curitiba 80210-170, Brazil

TEL: +55-41-99223-8274

E-mail: juliana.schussel24@gmail.com

ORCID: https://orcid.org/0000-0001-5204-0782

(c) This is an open-access article distributed under the terms of the Creative Commons Attribution Non-Commercial License (http://creativecommons.org/ licenses/by-nc/4.0/), which permits unrestricted non-commercial use, distribution, and reproduction in any medium, provided the original work is properly cited. Copyright (C) 2021 The Korean Association of Oral and Maxillofacial Surgeons. All rights reserved. lung cancer and manages lytic lesions in multiple myeloma ${ }^{2,3}$.

Medication-related osteonecrosis of the jaw (MRONJ) is a severe adverse reaction to antiresorptive drugs.(Fig. 1) This condition can affect both maxillary bones, with higher incidence in the mandible. The diagnosis is made when the patient was exposed, previously or currently, to antiresorptive or antiangiogenic agents and presents exposed bone that persists for more than eight weeks, without history of radiation therapy in the head and neck area ${ }^{4,5}$. A therapeutic protocol has not been established, and clinical resolution is difficult. Consequently, prevention is imperative to avoid this complication $^{4,6,7}$.

The aim of this study was to evaluate the profile of patients on antiresorptive therapies for cancer treatment and assess presence of oral lesions, oral hygiene status, and knowledge regarding MRONJ. 


\section{Materials and Methods}

This study was approved by the Research Ethics Committee of Erasto Gaertner Cancer Center under protocol (No. 65123116.1.0000.0098) and is indexed at the national database of research involving human subjects. The written informed consent was obtained from all patients.

This was an observational cross-sectional study that evaluated patients treated with antiresorptive agents, previously or currently. All patients were assigned to the Department of Oral and Maxillofacial Surgery at Erasto Gaertner Cancer Center, referred by the clinical oncologist of the same institu-

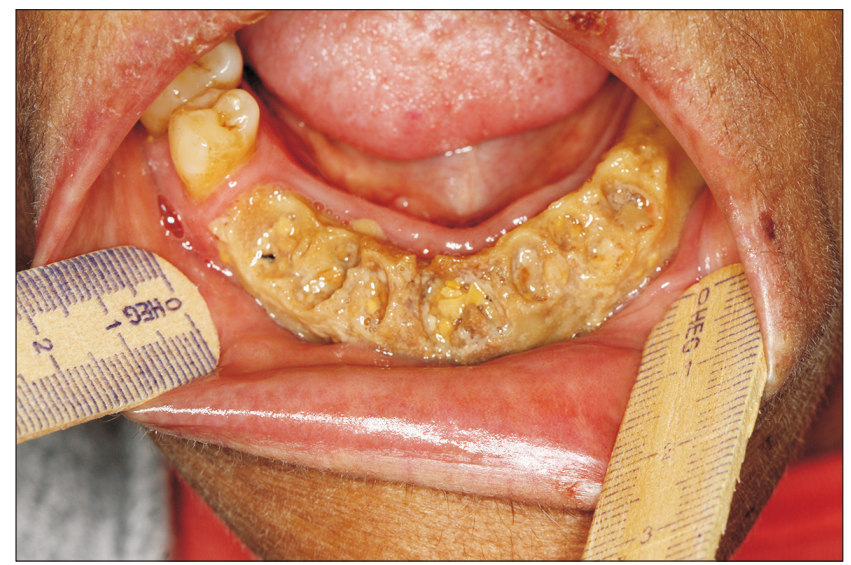

Fig. 1. Severe medication-related osteonecrosis of the jaw (MRONJ) in a patient with multiple myeloma who received pamidronate.

Vitor Hugo Candido Ferreira et al: Antiresorptive medication in oncology: the clinical and dental profile of patients in a reference center. J Korean Assoc Oral Maxillofac Surg 2021

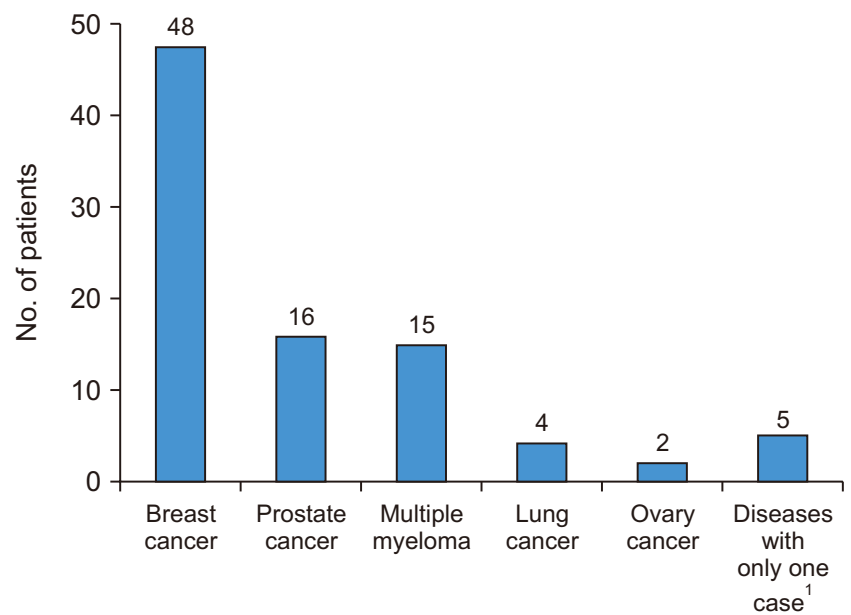

Fig. 2. Distribution of primary disease; ${ }^{1}$ uterine cancer, renal liposarcoma, ovarian cancer, and metastatic bone tumor with unknown primary location.

Vitor Hugo Candido Ferreira et al: Antiresorptive medication in oncology: the clinical and dental profile of patients in a reference center. J Korean Assoc Oral Maxillofac Surg 2021 tion.

The study comprised patients with malignant bone pathologies and bone metastases. The patients were either a candidate for use, currently using, or previously used antiresorptive drugs. The study excluded patients with oral metastatic or metaplastic lesions, grafts or flaps on the jaws, or who had undergone radiotherapy in the region.

Demographic and clinical data were collected on patient age at consultation, sex, oral hygiene, presence of oral disease/condition, type of drug and duration of use, and knowledge regarding the risks associated with drug use.

Team calibration was performed to conduct oral hygiene analysis based on the simplified oral hygiene index (IHO$\mathrm{S})^{8}$, with a score of 0 as good hygiene, 1 as regular, and 2 and 3 as poor. The presence of one or more residual roots, teeth with open cavities, or periodontal abscesses were categorized as poor hygiene. Patients with spontaneous gingival bleeding, plaque-related periodontal hyperplasia, and spontaneous gingival suppuration, whether or not associated with tooth mobility, were included in the clinical profile of periodontal disease.

\section{Results}

From July 2017 to December 2018, a total of 90 patients was referred to the outpatient clinic at the Department of Oral and Maxillofacial Surgery at Erasto Gaertner Cancer Center for oral evaluation to prevent osteonecrosis.

Among the 90 patients, 64 were female and 26 male (ratio, 2.5:1). The ages ranged from 29 to 87 years, with a mean of 61 years. The underlying diseases for referring patients for antiresorptive medication use are shown in Fig. 2.

The most commonly used antiresorptive drugs with po-

Table 1. Drug distribution, standard dose, and number of patients using the drugs

\begin{tabular}{lcc}
\hline \multicolumn{1}{c}{ Drug } & Dose (mg) & No. of patients ${ }^{3}$ \\
\hline Zolendronic acid IV & 4 & 70 \\
Trastuzumab IV & 440 & 4 \\
Pamidronate IV & 90 & 2 \\
Denosumab SC & 60 & 2 \\
Patients before medication start ${ }^{1}$ & - & 6 \\
Multiple drugs ${ }^{2}$ & - & 6 \\
\hline (IV: intravenous, SC: subcutaneous) & \\
${ }^{1}$ Patients evaluated prior to medication use. \\
${ }^{2}$ Associations of zoledronic acid and trastuzumab (3), zoledronic acid \\
and pamidronate (2), zoledronic acid and gefitinib (1). \\
${ }^{3}$ Six patients started medication after consultation. \\
Vitor Hugo Candido Ferreira et al: Antiresorptive medication in oncology: the clinical and \\
dental profile of patients in a reference center. J Korean Assoc Oral Maxillofac Surg 2021
\end{tabular}


tential risk of MRONJ were zoledronic acid, pamidronate, denosumab, trastuzumab, and gefitinib. Drug distribution, standard dose, and number of patients using the drugs are presented in Table 1.

Evaluation of oral cavity showed a high $30 \%$ prevalence of periodontal disease in the study cohort, followed by oral candidiasis (7.8\%) and proliferative lesions or vascular malformations $(4.5 \%)$.

MRONJ was diagnosed in nine patients (10.0\%); five were female with a mean age of 65 years. Two patients had no history of local trauma or dental procedures, and bone exposure was considered spontaneous. Five patients reported dental extraction, and the other two had experienced bone exposure associated with severe periodontal disease. Information related to MRONJ diagnosis is presented in Table 2.

After evaluation, $63.3 \%$ of the patients were categorized as regular or poor hygiene. Based on clinical and radiographic diagnosis (grade III mobility, furcation involvement, longitudinal dental fractures, unrestorable carious lesions, advanced periodontitis), $21 \%$ of the patients had indication for extraction. Among the patients diagnosed with MRONJ, five had active periodontal disease, and only one was categorized as good oral hygiene.

Regarding patient knowledge about the side effects of medication, $60 \%$ stated not being aware of the risks of MRONJ. The subjects also stated they were not familiar with the contraindications of dental surgery in patients with a history of antiresorptive or antiangiogenic therapy.

\section{Discussion}

Most studies on MRONJ focused on disease description and did not evaluate patient profile. As far as we know, this study presents the largest sample of patients using antiresorptive agents for cancer treatment in Latin America, describing the main characteristics of patients at risk of MRONJ.
MRONJ directly affects the quality of life of patients, leading to high morbidity. Due to the limited therapeutic options, treatments are not always resolutive ${ }^{6,9}$. Management can be challenging and usually requires a multidisciplinary approach, with a variety of interventions and care ${ }^{7}$ The indicated treatment is directly associated with disease stage and general health status ${ }^{4}$.

Previous studies have demonstrated that the route of drug administration is associated with increased risk for MRONJ development, with oral drugs having a lower incidence than intravenous (IV) medications ${ }^{5,10}$. Therefore, MRONJ development should be considered in cancer patients in whom drugs are almost exclusively administered IV.

Most patients in the present study are female, with a 2.5:1 ratio between female and male; these females had a mean age of 60 years. Breast and prostate cancer and multiple myeloma were the most frequent malignancies in the study. Zoledronic acid was the most frequently used medication, followed by pamidronate, trastuzumab, and denosumab. Zoledronic acid has a high relative potency compared with other medications and presents a higher risk of MRONJ. Use of this medication is increasing in cancer treatments due to its efficiency, implying a greater number of patients at risk of MRONJ development ${ }^{9}$. Therefore, those involved in disease treatment such as oncologist, patient, and other health professionals should be aware of the need for preventive actions and constant followup during and after use of antiresorptive drugs ${ }^{7}$.

The majority of patients treated at our hospital are from the public health system, are of low-income, and have difficulty accessing oral health services. Of them, $67 \%$ of the patients were classified as regular or poor oral hygiene. In previous studies, oral health was a risk factor for MRONJ, and preventive measures must include dental consultation ${ }^{4,5,11}$. Other risk factors include inflammatory dental diseases, dental caries, torus mandibularis and palatinus, and traumatic factors such as unadapted dental prostheses ${ }^{3}$. Inflammation and

Table 2. Clinical information of diagnosed medication-related osteonecrosis of the jaw

\begin{tabular}{|c|c|c|c|c|c|}
\hline Site & $\begin{array}{c}\text { Exposure size } \\
(\mathrm{mm})\end{array}$ & Stage & Disease & Medication & Dose (mg) \\
\hline Mandible & 25 & 3 & Breast & Denosumab & 1 \\
\hline Mandible & 20 & 2 & Breast & Zoledronic acid & 2 \\
\hline Maxilla & 10 & 2 & Breast & Zoledronic acid & 18 \\
\hline Mandible & 20 & 2 & Breast & Zoledronic acid & 1 \\
\hline Mandible & 5 & 2 & Multiple myeloma & Zoledronic acid & 12 \\
\hline Mandible & 5 & 2 & Prostate & Zoledronic acid & 1 \\
\hline Maxilla & 10 & 2 & Prostate & Zoledronic acid & 39 \\
\hline Maxilla & 10 & 2 & Multiple myeloma & Zoledronic acid & 6 \\
\hline Maxilla & 30 & 3 & Prostate & Zoledronic acid & 31 \\
\hline
\end{tabular}

Vitor Hugo Candido Ferreira et al: Antiresorptive medication in oncology: the clinical and dental profile of patients in a reference center. J Korean Assoc Oral Maxillofac Surg 2021 
bacterial infection are implicated in MRONJ pathogenesis ${ }^{12}$. Good oral hygiene that includes dental floss and mouthwash is an important measure to reduce risk of $\mathrm{MRONJ}^{5}$. Patient preparation must include extraction of compromised teeth, management of active carious lesions, pulp infections, and periodontal disease control. Most patients in the present study had their first consultation with a dentist after starting the medication, which reduced the chance of adequate oral preparation and exposed the patients to risk. On the first consultation, nine cases presented with MRONJ, and two of had no oral complaints, showing the need for preventive followup for early detection; other patients had undergone tooth extracted and were unaware of the risk of complications.

Patient knowledge regarding MRONJ is essential for prevention $^{6,7}$. More than half of the patients evaluated were unaware of the risk for this complication. The misconception of the actual effects of the drug, which is usually referred to as a vitamin or calcium supplement, leads to lack of preventive care.

MRONJ pathophysiology is apparently multifactorial, and inflammation or infection, trauma, and decreased bone turnover are associated with disease development ${ }^{1,4}$. Although spontaneous development has been described by some authors, in most reports, MRONJ is associated with tooth extraction due to pulp infection, periapical lesions, and bacterial infections ${ }^{8,13,14}$. This connection was observed in an animal study, indicating that such infections precede the appearance of necrotic bone $\mathrm{b}^{11,15,16}$.

\section{Conclusion}

Although preventive measures do not exclude the possibility of MRONJ, they have been shown to reduce the risk ${ }^{17,18}$. Preventive measures can be used to help potentially at-risk patients and should be encouraged. Identifying the patient profile and needs facilitates not only the preventive process, but also the emergence of new therapeutic options. Our study shows that most patients were weakened by metastatic disease, anti-neoplastic treatment, and by issues associated with aging because most subjects were over 60 years of age. Collectively, this information should be considered for management of preventive and therapeutic measures.

\section{ORCID}

Vitor Hugo Candido Ferreira, https://orcid.org/0000-00018385-6549
Aristilia Tahara Kemp, https://orcid.org/0000-0001-9992-3451

Joana Vendruscolo, https://orcid.org/0000-0001-5861-7730

Laurindo Moacir Sassi, https://orcid.org/0000-0002-9333-2498

Juliana Lucena Schussel, https://orcid.org/0000-0001-52040782

\section{Authors' Contributions}

V.H.C.F. participated in study design, data collection, statistical analysis and wrote the manuscript. A.T.K. participated in data collection, statistical analysis and helped to draft the manuscript. J.V. participated in data collection, statistical analysis and helped to draft the manuscript. L.M.S. participated in study design, data collection, and final review of the manuscript. J.L.S. participated in study design and coordination, data collection, statistical analysis and final review of the manuscript. All authors read and approved the final manuscript.

\section{Ethics Approval and Consent to Participate}

This study was approved by the Research Ethics Committee of Erasto Gaertner Cancer Center under protocol (No. 65123116.1.0000.0098), and the written informed consent was obtained from all patients.

\section{Conflict of Interest}

No potential conflict of interest relevant to this article was reported.

\section{References}

1. Nicolatou-Galitis O, Schiødt M, Mendes RA, Ripamonti C, Hope $\mathrm{S}$, Drudge-Coates L, et al. Medication-related osteonecrosis of the jaw: definition and best practice for prevention, diagnosis, and treatment. Oral Surg Oral Med Oral Pathol Oral Radiol 2019;127:117-35. https://doi.org/10.1016/j.oooo.2018.09.008

2. Coleman RE. Skeletal complications of malignancy. Cancer 1997;80(8 Suppl):1588-94. https://doi.org/10.1002/(sici)10970142(19971015)80:8+<1588::aid-cncr9>3.3.co;2-z

3. Şahin O, Odabaşı O, Aliyev T, Tatar B. Risk factors of medicationrelated osteonecrosis of the jaw: a retrospective study in a Turkish subpopulation. J Korean Assoc Oral Maxillofac Surg 2019;45:10815. https://doi.org/10.5125/jkaoms.2019.45.2.108

4. Ruggiero SL, Dodson TB, Fantasia J, Goodday R, Aghaloo T, Mehrotra B, et al. American Association of Oral and Maxillofacial Surgeons position paper on medication-related osteonecrosis of the jaw--2014 update. J Oral Maxillofac Surg 2014;72:1938-56. https://doi.org/10.1016/j.joms.2014.04.031

5. Nifosì AF, Zuccarello M, Nifosì L, Hervas Saus V, Nifosì G. Osteonecrosis of the jaw in the era of targeted therapy and immunotherapy in oncology. J Korean Assoc Oral Maxillofac Surg 
2019;45:3-8. https://doi.org/10.5125/jkaoms.2019.45.1.3

6. Al Abdullateef A, Alhareky MS. Awareness among patient at risk of developing Medication Related Osteonecrosis of the Jaw (MRONJ) - a primary prevention strategy. Saudi Pharm J 2020;28:771-8. https:// doi.org/10.1016/j.jsps.2020.05.004

7. Sturrock A, Preshaw PM, Hayes C, Wilkes S. Perceptions and attitudes of patients towards medication-related osteonecrosis of the jaw (MRONJ): a qualitative study in England. BMJ Open 2019;9:e024376. https://doi.org/10.1136/bmjopen-2018-024376

8. Greene JC, Vermillion JR. The simplified oral hygiene index. J Am Dent Assoc 1964;68:7-13. https://doi.org/10.14219/jada.archive.1964.0034

9. Patel V, Kelleher M, Sproat C, Kwok J, McGurk M. New cancer therapies and jaw necrosis. Br Dent J 2015;219:203-7. https://doi. org/10.1038/sj.bdj.2015.680

10. Heufelder MJ, Hendricks J, Remmerbach T, Frerich B, Hemprich A, Wilde F. Principles of oral surgery for prevention of bisphosphonate-related osteonecrosis of the jaw. Oral Surg Oral Med Oral Pathol Oral Radiol 2014;117:e429-35. https://doi.org/10.1016/ j.oooo.2012.08.442

11. Tsao C, Darby I, Ebeling PR, Walsh K, O’Brien-Simpson N, Reynolds E, et al. Oral health risk factors for bisphosphonate-associated jaw osteonecrosis. J Oral Maxillofac Surg 2013;71:1360-6. https:// doi.org/10.1016/j.joms.2013.02.016

12. Sandhu S, Salous MH, Sankar V, Margalit DN, Villa A. Osteonecrosis of the jaw and dental extractions: a single-center experience. Oral Surg Oral Med Oral Pathol Oral Radiol 2020;130:515-21. https://doi.org/10.1016/j.0ooo.2020.07.001

13. Poubel VLDN, Silva CAB, Mezzomo LAM, De Luca Canto G, Rivero ERC. The risk of osteonecrosis on alveolar healing after tooth extraction and systemic administration of antiresorptive drugs in rodents: a systematic review. J Craniomaxillofac Surg 2018;46:245-56. https://doi.org/10.1016/j.jcms.2017.11.008

14. Landesberg R, Woo V, Cremers S, Cozin M, Marolt D, Vunjak-
Novakovic G, et al. Potential pathophysiological mechanisms in osteonecrosis of the jaw. Ann N Y Acad Sci 2011;1218:62-79. https://doi.org/10.1111/j.1749-6632.2010.05835.x

15. Gaudin E, Seidel L, Bacevic M, Rompen E, Lambert F. Occurrence and risk indicators of medication-related osteonecrosis of the jaw after dental extraction: a systematic review and meta-analysis. J Clin Periodontol 2015;42:922-32. https://doi.org/10.1111/ jcpe. 12455

16. Tsurushima H, Kokuryo S, Sakaguchi O, Tanaka J, Tominaga K. Bacterial promotion of bisphosphonate-induced osteonecrosis in Wistar rats. Int J Oral Maxillofac Surg 2013;42:1481-7. https://doi. org/10.1016/j.ijom.2013.06.011

17. Dimopoulos MA, Kastritis E, Bamia C, Melakopoulos I, Gika D, Roussou M, et al. Reduction of osteonecrosis of the jaw (ONJ) after implementation of preventive measures in patients with multiple myeloma treated with zoledronic acid. Ann Oncol 2009;20:117-20. https://doi.org/10.1093/annonc/mdn554

18. Bramati A, Girelli S, Farina G, Dazzani MC, Torri V, Moretti A, et al. Prospective, mono-institutional study of the impact of a systematic prevention program on incidence and outcome of osteonecrosis of the jaw in patients treated with bisphosphonates for bone metastases. J Bone Miner Metab 2015;33:119-24. https://doi. org/10.1007/s00774-014-0566-x

How to cite this article: Ferreira VHC, Kemp AT, Vendruscolo J, Sassi LM, Schussel JL. Antiresorptive medication in oncology: the clinical and dental profile of patients in a reference center. J Korean Assoc Oral Maxillofac Surg 2021;47:20-24. https://doi. org/10.5125/jkaoms.2021.47.1.20 